\title{
Parlementaire et saint laïque. La mort publique de Carlo Poerio (Italie, 1867)
}

\author{
Pierre-Marie DELPU \\ Membre scientifique, École des hautes études hispaniques et ibériques \\ pierre-marie.delpu arobase casadevelazquez.org
}

Le 28 avril 1867, Carlo Poerio, alors député du Royaume d'Italie, meurt à Florence, frappé d'une pneumonie. Les célébrations que des acteurs publics organisent en son honneur, dans les jours qui ont suivi son décès, engagent une mythification précoce qui l'envisage comme un martyr politique. Ce statut renvoie aux souffrances qu'il aurait subies au titre de son engagement politique, et auquel la fonction de parlementaire, qu'il a occupée de manière ininterrompue depuis 1860, aurait contribué de façon décisive. Poerio présente en effet un parcours de patriote exemplaire, qui s'intègre dans la propagande du nouvel État italien en quête de figures fondatrices, capables de légitimer son existence politique contestée. Né en 1803 à Naples, issu d'une famille de la noblesse calabraise, il a connu plusieurs expériences d'exil puis d'emprisonnement, a tenté d'unifier les oppositions de gauche à la monarchie des Bourbons dans les années 1840 avant d'être ministre de l'Instruction publique du bref gouvernement constitutionnel napolitain en 1848. Emprisonné à nouveau, en 1849, à la suite d'un procès pour conspiration, sa détention est ensuite largement médiatisée : jusqu'à son amnistie en janvier 1859, il est devenu, temporairement, l'un des protagonistes de l'actualité politique européenne, alors que la presse libérale lui prête un statut de martyr, décrivant abondamment les souffrances qu'il a subies pendant son séjour prolongé en prison'1.

La requalification de Poerio en martyr de l'italianité, développée dans les jours qui ont suivi sa mort, s'appuie sur des ressorts différents. Elle fait évidemment référence à sa détention passée, en la replaçant dans une relecture plus large de sa biographie, réévaluée sous l'angle du martyre. Les hommages funèbres qui lui sont consacrés vont dans ce sens. Ils témoignent d'une perception renouvelée du martyre politique : d'abord limitée à la gauche patriotique, qui en faisait un outil de la résistance aux monarchies italiennes préunitaires, la notion est devenue, depuis 1860, un outil central de la religion civile élaborée par les classes dirigeantes du nouvel État. Elle fournit en effet un modèle moral, auquel les masses italiennes chercheraient à s'identifier et qu'elles pourraient imiter. Il devient ainsi un outil fondamental de la pédagogie nationale italienne, ancré dans une ample culture de la mort publique qui a d'abord été clandestine avant de devenir progressivement officielle ${ }^{2}$. Les hommages rendus à Poerio dans les jours qui ont

\footnotetext{
${ }^{1}$ Sur l'itinéraire médiatique de Carlo Poerio, voir Pierre-Marie Delpu, L'affaire Poerio. La fabrique d'un martyr révolutionnaire européen (1851-1859), Paris, CNRS Éditions, 2021. 2 Lucy J. Riall, "Martyr Cults in Nineteenth-Century Italy », The Journal of Modern History, $\mathrm{n}^{\circ} 82 / 2,2010$, p. 255-287. Sur les liturgies civiles, voir Fulvio Conti, Italia immaginata. Sentimenti, memoria e politica fra Otto e Novecento, Pise, Pacini, 2017. Sur la mort publique, Silvia Cavicchioli, I Cimeli della patria. Politiche della memoria nel lungo Ottocento, Rome, Carocci, 2021.
} 
immédiatement suivi sa mort en font partie : orchestrés par l'État, ils transforment une icône internationale de la résistance à l'oppression en une figure de l'italianité politique. La mise en œuvre des funérailles nationales en son honneur, les constructions politiques qui entourent son cadavre, les récits du martyre sont révélateurs de cette mutation, identifiant Carlo Poerio aux formes laïques de la sainteté.

\section{L'ORGANISATION DES FUNERAILLES PUBLIQUES}

Les obsèques officielles de Carlo Poerio, organisées à l'initiative de la Chambre des Députés, transforment un deuil privé en un événement d'ampleur nationale. La session parlementaire du 29 avril 1867, au lendemain de sa mort, constitue le point de départ des hommages collectifs qui lui sont rendus. À l'obituaire prononcé par le président de la Chambre, l'avocat florentin Adriano Mari, succèdent en effet des éloges prononcés par des parlementaires issus de l'entourage immédiat du défunt. Francesco Crispi, Sicilien, ancien républicain rallié depuis peu à la monarchie, Giuseppe Pisanelli, député des Pouilles de tendance modérée, qui a coporté avec Poerio, en 1862, un projet de loi pour l'abolition de la peine de mort, et Mariano D’Ayala, libéral napolitain, font ainsi partie des principaux orateurs ${ }^{3}$. C'est à ce dernier, auteur de martyrologes politiques très nombreux tout au long des années 1850 et 1860, que l'on doit l'initiative de funérailles publiques, immédiatement acceptée à l'unanimité des présents ${ }^{4}$. Le poids des milieux parlementaires a donc été déterminant dans la mise en œuvre des hommages : plusieurs représentants accompagnent la suite du processus, prononcent ou publient des obituaires, ou assistent aux obsèques. C'est le cas d'Enrico Fossombroni, député d'Arezzo, qui représente la Chambre auprès du conseil communal de Florence, ou des Méridionaux Filippo Abignente et Giuseppe Massari qui accompagnent la dépouille de Poerio à Naples, en vue de son inhumation ${ }^{5}$. Le rôle joué par les députés contribue donc de façon décisive à la mise en scène publique de la mort de Poerio, qui s'inscrit dans un processus plus large de sécularisation et de laïcisation du deuil, que l'on soustrait à la fois à la famille et à l'Église $^{6}$. Il a contribué, depuis la fin du XVIII ${ }^{\mathrm{e}}$ siècle, à la célébration croissante des « grands hommes » valorisés par les constructions partisanes et nationales, dont ils ont été des instruments de légitimation.

$\mathrm{Au}$ milieu du XIX ${ }^{\mathrm{e}}$ siècle, l'utilisation de plus en plus grande de l'espace médiatique

\footnotetext{
${ }^{3}$ Archivio Storico della Camera dei Deputati (ASCD), Discussioni, 29 avril 1867.

${ }^{4}$ Parmi une œuvre importante, voir particulièrement, outre les notices consacrées à des martyrs napolitains dans le Panteon dei martiri della libertà italiana (Turin, Fontana, 1851-1852), I nostri morti in Napoli e Sicilia : statistica politica, Naples, G. Nobile, 1860, et I primi quattro martiri della libertà italiana nel 1821, Naples, Lombardi, 1861.

5 Outre ASCD, Discussioni, 29 avril 1867, voir aussi l'opuscule publié à l'initiative du Conseil communal de Naples, Carlo Poerio, Naples, Consiglio comunale, 1867, qui rassemble les textes lus ou rédigés par des élus en l'honneur de Poerio.

${ }^{6}$ Fulvio Conti, «L'Urne dei forti. Religioni politiche e liturgie funebri nei secoli XIX e XX », dans Maurizio Ridolfi (dir.), Rituali civili. Storie nazionali e memorie pubbliche nell'Europa contemporanea, Rome, Gangemi, 2006, p. 115-134.
} 
par les cultures de la célébrité explique que cette évolution s'amplifie. Elle consacre des acteurs jugés représentatifs, érigés en figures d'autorité destinées à étayer des mouvements politiques ${ }^{7}$. L'Italie post-unitaire recourt à ce processus intégrateur, autour de panthéons hétéroclites qui associent des détenteurs de l'autorité politique, des combattants, d'anciens exilés, qualifiés de façon souvent indistincte de " héros » ou de «martyrs ». Le statut de grand homme prêté à Carlo Poerio relève de ce dispositif mémoriel, qui combine ses différentes identités sociales. Ses thuriféraires le présentent à la fois comme ancien avocat, ancien protagoniste du mouvement libéral napolitain, ancien martyr vivant de la cause des libertés et ancien député du Royaume d'Italie. Pour Abignente, député méridional et ami du défunt, son statut d'homme public justifie amplement que des hommages nationaux lui soient rendus, financés sur les dépenses publiques. Lors des obsèques célébrées à Naples, le 3 mai 1867, il rappelle que la nouvelle de sa mort «a tout de suite été perçue comme une mésaventure publique » : l'ampleur nationale de l'événement et la nécessité de célébrer la mort de Poerio ont fait consensus entre des factions parlementaires jusque-là très divisées autour de la forme à donner au nouvel État italien ${ }^{8}$.

Mort à Florence, alors capitale du royaume d'Italie depuis 1865, Carlo Poerio a été inhumé à Naples : la translation du corps et l'organisation des obsèques ont impliqué les conseils communaux des deux villes, tous deux compétents en matière de gestion des pompes funèbres ${ }^{9}$. Lors de sa séance du 30 avril 1867, informée par télégramme du décès de Poerio, la mairie de Naples demande à celle de Florence de récupérer la dépouille du défunt pour pouvoir organiser des funérailles publiques dans sa ville natale, dont il a par ailleurs été député pendant sept ans ${ }^{10}$. Un premier hommage est en effet rendu, peu après l'annonce des funérailles publiques, par le conseil communal de Florence, où siège notamment Antonio Scialoja, libéral napolitain devenu sénateur du Royaume d'Italie. La ville de Florence le mandate pour la représenter auprès de la mairie de Naples, pour suivre le convoi funèbre et prononcer une oraison au nom des instances municipales florentines. Son origine méridionale et son amitié pour Poerio constituent les principaux arguments mobilisés, qu'il relaie auprès du public napolitain, dans la basilique où sont célébrées les obsèques ${ }^{11}$. Scialoja joue donc le rôle d'un intercesseur, chargé d'accompagner le convoi funèbre jusqu'à l'inhumation. Après cette dernière, le conseil municipal de Naples mandate à son tour l'un de ses membres, Giuseppe Capitelli, pour exprimer la reconnaissance de la commune à la ville de Florence. Dans un discours

\footnotetext{
${ }^{7}$ Edward Berenson, Eva Giloi (dir.), Constructing Charisma. Celebrity, Fame and Power in Nineteenth-Century Europe, New York, Berghahn books, 2010.

${ }^{8}$ Parole dette dal deputato Filippo Abignente nella basilica di S. Francesco di Paola, 3 mai 1867, citées dans Carlo Poerio..., op. cit., p. 18.

9 Pour Naples, voir Diego Carnevale, L'affare dei morti. Mercato funerario, politica e gestione della sepoltura a Napoli (secoli XVII-XIX), Rome, École française de Rome, 2014. ${ }^{10}$ Archivio Storico Municipale di Napoli (ASMN), Atti del Consiglio Comunale, vol. VII, p. 59, session du 30 avril 1867.

11 Discorso pronunciato dal senatore Antonio Scialoja nella basilica di S. Francesco di Paola, 3 mai 1867, cité ibid., p. 6.
} 
immédiatement publié dans le journal florentin L'Opinione, le 9 mai 1867, il exprime « le sentiment de reconnaissance des Napolitains pour le témoignage d'honneur rendu en mémoire du regretté Poerio ${ }^{12}$ ». Fin mai 1867, le maire de Naples Fedele De Siervo se rend à son tour à Florence, pour représenter lui-même le conseil municipal qu'il dirige lors de la cérémonie que la capitale du royaume organise alors en l'honneur de Carlo Poerio ${ }^{13}$.

À côté de ces commémorations officielles, des initiatives privées plus diffuses apparaissent tout au long du mois de mai 1867. C'est surtout dans les milieux savants, premiers soutiens du mouvement libéral à Naples, que des acteurs privés évoquent son souvenir. À Florence, l'homme de lettres napolitain Saverio Baldacchini, alors député élu dans les Pouilles, prononce ainsi une oraison funèbre auprès des membres de la Reale Accademia di Archeologia, Letteratura e Belle Arti, l'une des principales académies de belles-lettres du nouvel État, le 14 mai 1867. Soucieux de s'inscrire dans la ligne dominante du pouvoir italien, qui cherche à donner au royaume un panthéon légitimateur de héros et de martyrs, il rattache Poerio à la première catégorie, " ceux qui se sacrifient pour les autres, et non ceux qui sacrifient les autres pour eux-mêmes ${ }^{14}$ ». Des hommages comparables interviennent dans les milieux universitaires, particulièrement à Naples, afin de célébrer les qualités de juriste de Carlo Poerio. Ils sont d'abord l'initiative d'un étudiant en dernière année de droit, Raffaele De Cesare, désireux de rendre hommage à son professeur Luigi Settembrini, ami proche de Poerio qu'il a côtoyé en prison dans les années 1850. Lu en chaire le 7 mai 1867, soit quatre jours après les obsèques, le discours est publié le lendemain dans le Giornale di Napoli puis réédité sous la forme d'un opuscule ${ }^{15}$. Un mois et demi plus tard, Settembrini lui-même fait un éloge funèbre de Poerio face aux étudiants de la faculté de droit de Naples, qu'il invite à suivre son exemple ${ }^{16}$. Ces initiatives récurrentes révèlent la capacité d'organisation d'acteurs publics et privés pour célébrer la mémoire d'une figure jugée fondatrice et exemplaire. Les traitements du cadavre du défunt montrent sa transposition en corps politique, exposé au public florentin et napolitain.

\section{LE CORPS DE POERIO, CADAVRE ET RELIQUE}

Les traitements accordés à la dépouille de Poerio montrent son édification en objet politique digne d'être commémoré, qu'une partie des observateurs qualifie d'ailleurs de

\footnotetext{
12 L'Opinione, 9 mai 1867, p. 1.

13 ASMN, Atti del Consiglio Comunale, vol. VIII, p. 2, session du 28 mai 1867.

14 Della vita e dei tempi di Carlo Poerio: discorso recitato nella reale Accademia di Archeologia Letteratura e Belle Arti nella tornata del di 14 maggio 1867 dal socio ordinario Saverio Baldacchini, Naples, Stamperia della Reale Università, 1867, p. 3. La citation, placée en exergue de l'ouvrage, est attribuée à Massimo D'Azeglio, ancien Premier ministre du Royaume de Piémont-Sardaigne au début des années 1850.

15 Raffaele De Cesare, Carlo Poerio, Naples, s.n., 1867.

${ }^{16}$ Luigi Settembrini, In morte di Carlo Poerio. Discorso, Naples, Tip. del Giornale di Napoli, 1867.
} 
« relique », par référence au modèle chrétien de la sainteté ${ }^{17}$. Cette qualification relève d'un processus de sacralisation courant dans les sociétés du XIX siècle, qui a permis d'affirmer l'exceptionnalité d'acteurs politiques autour de leurs actions exemplaires et de leurs qualités morales. Dans le cas de Carlo Poerio, il s'appuie sur les expériences récurrentes du martyre qui lui sont prêtées, qu'il s'agisse des stigmates de son emprisonnement prolongé dans les années 1850 ou de sa déchéance physique alors qu'il était député du royaume d'Italie. Pour Filippo Abignenti, par exemple, le port des chaînes qui lui a été imposé en détention pendant près de dix ans, les déplacements forcés qu'il a subis, les phases d'exil expliquent l'état du cadavre, qui lui paraît « prostré ». Ferdinando Fonseca et Mariano D'Ayala, qui ont assisté à l'agonie du député, confirment cet état. En décrivant ainsi l'affaiblissement de Poerio, tous en font le reflet des souffrances qu'il a subies, rejoignant ainsi le sens premier de la catégorie « martyr », définie par sa fonction de témoignage ${ }^{18}$.

$\mathrm{Au}$ lendemain de sa mort, le corps de Poerio fait l'objet d'une préparation préinhumatoire, encadrée par le conseil communal de Florence et par plusieurs parlementaires, notamment méridionaux, dont Fonseca, Crispi et Scialoja, qui cosignent avec d'autres députés les procès-verbaux préalables aux traitements appliqués au cadavre. Celui-ci est d'abord embaumé, selon une pratique alors courante chez les élites européennes du temps ; le cœur du défunt est ensuite extrait du corps, placé dans un vase de cristal pour exposer ce « héros de souffrances » - expression selon laquelle le procèsverbal le caractérise - à la "vénération universelle ${ }^{19}$ ». Effectuée par deux médecins proches du courant modéré auquel appartenait Poerio, Giuseppe Barellai et Cosimo Franchetti, qui ont tous deux occupé des charges municipales et législatives, la manipulation contribue à transformer le corps du défunt en un corps politique, ancré dans la propagande nationale du royaume d'Italie. Le lendemain, une délégation de parlementaires se recueille auprès de la dépouille, sur proposition de D'Ayala ${ }^{20}$. Par la suite, le cadavre est clôturé dans une caisse mortuaire scellée de la mairie de Florence, afin de pouvoir être transporté à Naples en vue de son inhumation ${ }^{21}$.

Le convoi funèbre relie ainsi le lieu de la mort de Poerio à sa ville d'origine qui était aussi son fief électoral. Il constitue une exception à la législation funèbre italienne qui limite le transport des cadavres à une zone restreinte, l'exclut autant que possible de la voie publique et impose qu'il ne se fasse que très tard dans la nuit, pour des raisons

17 Voir par exemple Discorso pronunciato dal senatore Antonio Scialoja nella basilica di S. Francesco di Paola, 3 mai 1867, cité. Sur la sacralité laïque, voir notamment Albrecht Burkardt, Jérôme Grévy (dir.), Reliques politiques, Rennes, Presses Universitaires de Rennes, 2020.

18 L'un et l'autre des discours sont reproduits dans Carlo Poerio, op. cit.

${ }^{19}$ Le procès-verbal pour l'embaumement de Poerio est signé par la mairie de Florence, le 29 avril 1867. Il est reproduit dans Carlo Poerio, op. cit., p. 110-111. Sur la pratique de l'embaumement au XIX ${ }^{\mathrm{e}}$ siècle, voir Anne Carol, L'embaumement, une passion romantique. France, XIXe siècle, Ceyzérieu, Champ Vallon, 2015.

${ }^{20}$ ASCD, Discussioni, 29 avril 1867.

21 Voir le procès-verbal signé par la mairie de Florence, le 30 avril 1867, cité dans Carlo Poerio, op. cit., p. 112. 
d'hygiène ${ }^{22}$. Le statut de grand homme déjà prêté au défunt justifie cet écart. Le corps et le cœur de Poerio sont ainsi acheminés par train, accompagnés d'une double délégation à la fois parlementaire et municipale, dirigée par Antonio Scialoja. Le cortège funèbre met en scène la célébrité du défunt, à travers le discours public prononcé dans la gare de Florence par Giuseppe Massari, Napolitain qui a participé au régime révolutionnaire de 1848 aux côtés de Poerio et est par la suite devenu député du royaume d'Italie ${ }^{23}$. Constituée d'anciens libéraux méridionaux qui ont accompagné le parcours politique de Carlo Poerio et occupent tous des charges parlementaires depuis l'unification de l'Italie, elle est chargée d'accompagner la dépouille jusqu'à sa remise à la mairie de Naples, en attente de son inhumation, intervenue le 3 mai 1867.

Célébrées à Naples, dans la basilique Saint-François de Paule, les obsèques témoignent d'un transfert de sacralité : l'ancien sanctuaire des Bourbons, dans lequel des révolutionnaires envisageaient d'installer le Parlement napolitain lors des insurrections de 1820 et 1848, accueille la cérémonie. En complétant un processus de sacralisation précédemment amorcé, elle s'inscrit dans la religion laïque destinée à renforcer l'identification des masses au nouvel État italien. La mise en scène du cadavre s'inscrit en effet dans une pédagogie politique qui trouve son point de départ avec les révolutions de la fin du XVIII siècle, où elle s'est particulièrement appliquée aux parlementaires : elle recourt à l'exposition publique des corps des défunts, dont on rappelle les blessures, visibles ou non, et la façon dont ils ont été suppliciés ${ }^{24}$.

Les discours prononcés à l'occasion des obsèques par des membres de la délégation parlementaire et du conseil municipal de Naples s'attachent à souligner la sacralité du corps exposé au public. L'un des adjoints de la mairie de Naples, Guglielmo Capitelli, affirme avoir sollicité auprès de la mairie de Florence ce qu'il qualifie de "relique», emblématique à la fois des combats menés par Poerio lui-même et du patriotisme italien en général, dont il serait représentatif 25 . Baldacchini, quant à lui, mentionne «des honneurs semblables à ceux qu'on rend aux princes, et tels que peu de princes reçoivent une telle reconnaissance spontanée de la part de leurs peuples ${ }^{26}$ ». À la suite de ces éloges mortuaires, le corps est provisoirement placé dans la tombe familiale du maire Felice De Siervo, dans l'attente d'une réinhumation qui n'intervient que plus tard ${ }^{27}$. Tous ces hommages, les éloges prononcés tout au long du mois de mai 1867 le montrent, visent moins à célébrer la mémoire du mort lui-même qu'à transmettre son exemple aux

\footnotetext{
22 Dino Mengozzi, Garibaldi taumaturgo. Reliquie laiche e politica nell'Ottocento, Manduria, Lacaita, 2008, p. 71.

${ }^{23}$ Parole dette in morte del deputato Poerio alla Camera dei Deputati il 29 aprile 1867 ed alla stazione della ferrovia per Napoli il giorno successivo, Florence, Eredi Botta, 1867.

${ }^{24}$ Michel Biard, La liberté ou la mort. Mourir en député, 1792-1795, Paris, Taillandier, 2015, chapitre 8.

25 Propos cités dans le journal florentin L'Opinione, 9 mai 1867, p. 2.

26 Della vita e dei tempi di Carlo Poerio..., op. cit., p. 9.

27 Procès-verbal du conseil communal de Naples, 3 mai 1867, cité dans Carlo Poerio, op. cit., p. 111.
} 
contemporains, au vu du caractère emblématique de son parcours de patriote ${ }^{28}$. Devant les étudiants de droit de la faculté de Naples, Luigi Settembrini rappelle que Poerio constitue un exemple à imiter au titre des souffrances qu'il a subies, dont le cadavre serait le reflet. Il invite son auditoire à l'imiter, et l'exhorte à « souffr[ir] simplement comme lui », pour s'assurer de "splendides compensations, des funérailles publiques et une statue $^{29} »$. La mise en scène du cadavre et les usages qui en sont faits sont donc représentatifs de la religion civile portée par les classes dirigeantes italiennes des lendemains de l'Unité : le corps de Poerio constitue une "relique sans miracle», représentative d'un parcours politique entièrement relu au prisme du martyre ${ }^{30}$.

\section{REDEFINIR LE MARTYRE}

Tous les éloges funèbres prononcés en l'honneur du défunt s'accordent à lui reconnaître les caractéristiques du martyr politique. Ces dernières ne sont formalisées par aucun texte théorique, mais correspondent à un ensemble d'expériences et de valeurs exprimées à la fois par une abondante littérature mémorielle et par des pratiques sociales convergentes, et sont identifiées sans difficulté par les contemporains ${ }^{31}$.

Le martyre politique, à l'instar de son équivalent religieux, est le produit d'une représentation qui reconstruit la vie et la mort de l'acteur concerné, afin d'en faire un modèle destiné au corps social ${ }^{32}$. Le récit du martyre recompose donc la logique biographique : la trajectoire de Poerio est ainsi réenvisagée de manière linéaire, comme une succession d'étapes d'un martyre quasi-permanent. Ils s'attachent ainsi à présenter Poerio en homme de douleur, image déjà développée dans la presse européenne des années 1850 qui décrivait abondamment les maladies qu'il avait contractées en prison, parce qu'elles auraient aggravé les difficultés de sa condition de détenu ${ }^{33}$. Dans le prolongement de ce suivi, l'inventaire des souffrances de Carlo Poerio constitue un trait commun à l'ensemble des textes d'éloge. Sur le modèle des hagiographies religieuses, que les martyrologes patriotiques des années 1840 et 1850 ont transféré au domaine politique, les orateurs rappellent la naissance noble du défunt, son enfance, le parcours politique de son père Giuseppe, figure du courant libéral modéré des Deux-Siciles. L'évocation des exils successifs que Poerio a connus, des persécutions engagées contre lui par les Bourbons, de son engagement au service du patriotisme napolitain et italien

28 Sur le discours de l'exemple, voir Arianna Arisi Rota, «Eroi, martiri, concittadini patriotti. I necrologi come pedagogia del ricordo », dans Arianna Arisi Rota, Monica Ferrari, Marco Morandi (dir.), Patrioti si diventa. Luoghi e linguaggi di pedagogia patriottica nell'Italia unita, Milan, FrancoAngeli, 2009, p. 143-156.

${ }^{29}$ L. Settembrini, In morte di Carlo Poerio..., op. cit., p. 27.

30 D. Mengozzi, Garibaldi taumaturgo..., op. cit., p. 31.

${ }^{31}$ L.J. Riall, « Martyr Cults in Nineteenth Century Italy », art. cité.

32 Parmi une bibliographie nombreuse consacrée au Moyen Âge et à l'époque moderne, voir notamment Maïté Billoré, Gilles Lecuppre (dir.), Martyrs politiques ( $X^{e}-X V^{e}$ siècle). Du sacrifice à la récupération partisane, Rennes, Presses Universitaires de Rennes, 2019.

33 P.-M. Delpu, L'Affaire Poerio..., op. cit., chapitre 5. 
traduisent la continuité des expériences de la souffrance. Pour Baldacchini, qui les regroupe sous le terme générique «martyre», elles ont pour fonction de préparer la vie posthume du défunt, qu'il qualifie de «triomphe ${ }^{34} »$.

En rapportant ainsi la biographie de Poerio à des modèles de célébration prédéfinis, les orateurs la reconstruisent au prisme de catégories émotionnelles qui mettent l'accent sur ses souffrances répétées, au détriment de la chronologie de son parcours. Pironti, par exemple, associe le sort que Poerio a subi en prison avec plusieurs des désillusions qu'il a connues dans son parcours de parlementaire aux lendemains de l'Unité. Il juxtapose par exemple son échec aux élections législatives de 1865 dans la $4{ }^{\text {ème }}$ circonscription de Naples, l'accueil qu'il a reçu du peuple britannique lors de son amnistie et de son exil en 1859, les douleurs liées au port permanent des chaînes pendant sa détention ${ }^{35}$. Le fait que ces étapes de sa biographie soient mentionnées sans chronologie ni contextualisation montre que la mémoire de Poerio est d'abord émotionnelle, dans la continuité des constructions médiatiques qui ont permis sa célébrité européenne dans les années 1850. Dès lors, les évocations relèvent moins du nécrologe classique que du martyrologe, destiné à montrer la force morale et l'exemplarité du défunt. D'autres lui prêtent des caractères héroïques, au travers de références issues d'un imaginaire guerrier hérité des premières décennies du XIX ${ }^{\mathrm{e}}$ siècle et réactivé au début des années 1860 avec le poids international du mythe garibaldien ${ }^{36}$. Celui-ci voit dans la figure du héros de guerre l'un des principaux modèles du martyr politique, alors valorisé dans une importante littérature de célébration à laquelle les publications de Mariano D’Ayala ont amplement contribué.

Les réécritures du martyre transfèrent donc sur les souffrances de Carlo Poerio des modèles extérieurs, qui identifient la constance morale à des qualités héroïques. La majorité des orateurs insistent à ce titre sur l'emprisonnement de Poerio. Plusieurs d'entre eux, comme Adriano Mari, le replacent dans la continuité d'expériences antérieures. Détenu à plusieurs reprises après les insurrections qui ont agité le Royaume des Deux-Siciles en 1837, 1844 et 1847, Poerio aurait surtout souffert de sa détention prolongée pendant les années $1850^{37}$. D'autres rappellent, à l'image de Raffaele De Cesare, à quel point cette expérience aurait été conditionnée par son exposition permanente à la violence politique. Sans qu'il soit possible de vérifier si l'information est avérée, il signale par exemple qu'emprisonné en Calabre en 1844, il aurait assisté, depuis sa prison, à l'exécution de plusieurs des protagonistes de la révolte locale ${ }^{38}$. Dès lors, le parcours de Poerio est présenté comme un engagement permanent en faveur de la liberté, déterminé par les pratiques de maintien de l'ordre dont il a été à la fois le spectateur et la victime. En replaçant son parcours de parlementaire dans le prolongement de ses expériences politiques passées, les auteurs d'éloges mortuaires les intègrent pleinement à son parcours de martyr. Saverio Baldacchini, par exemple, insiste sur son échec électoral aux

\footnotetext{
${ }^{34}$ Della vita e dei tempi di Carlo Poerio..., op. cit., p. 21.

35 Discours de Michele Pironti aux obsèques de Carlo Poerio, 3 mai 1867, op. cit.

${ }^{36}$ Lucy J. Riall, Garibaldi. Invention of a Hero, New Haven, Yale University Press, 2007.

37 ASCD, Discussioni, 29 avril 1867.

${ }^{38}$ R. De Cesare, Carlo Poerio..., op. cit., p. 11.
} 
élections législatives de 1865 : Poerio aurait été la victime collatérale de la corruption et du clientélisme politique, relayées par la presse de l'époque, principales raisons invoquées pour justifier sa défaite ${ }^{39}$.

La mort de Poerio constitue l'aboutissement de ce parcours, bien que les notations qu'elles suscitent soient limitées à quelques commentateurs, qui s'attachent à en retracer les circonstances. Mariano D'Ayala rappelle ainsi qu'elle ne doit pas être réduite à l'agonie physique consécutive à la pneumonie qu'il a contractée dans la semaine qui a précédé sa mort. Le parcours politique contrarié de sa famille, présenté comme un engagement constant au service de la patrie italienne, lui semble constituer un signe de «la consomption invisible de ses forces vitales». Les «tourments infinis qui consumaient sa vie », d'après D’Ayala, auraient été dissimulés aux contemporains par la grande modestie dont Poerio aurait fait preuve. En attribuant la mort du député à ces circonstances, il transforme donc une mort naturelle en une agonie patriotique, image de plus en plus utilisée dans le répertoire patriotique italien ${ }^{40}$. Il interroge aussi et surtout le statut du défunt, à qui il prête certaines des caractéristiques de la sainteté dans la continuité de textes d'éloge alors nombreux, qui le rapprochent de modèles apostoliques, voire christiques.

\section{MARTYR, Christ, PROPHETE ?}

Les caractères moraux que mettent en évidence les hommages publics rendus à Carlo Poerio montrent les évolutions de son existence symbolique. Longtemps présenté comme un martyr de la cause libérale, on lui prêtait la vertu politique revendiquée par les discours patriotiques du Risorgimento, en insistant sur sa force d'âme, sa capacité d'abnégation et de résignation, qui faisaient de lui un exemple digne d'être imité par ses contemporains, invités à déplorer et à venger le sort injuste qui lui était fait. Les discours produits à l'occasion de la mort de Poerio, à l'inverse, le rapprochent de figures empruntées à l'univers biblique, sous une forme sécularisée qui les réduit à leur fonction instrumentale. Plusieurs observateurs, d'abord, s'accordent à reconnaître son humilité et rappellent qu'il est mort pauvre, en dépit du patrimoine foncier dont disposait sa famille. La spoliation de ce dernier dans les années 1850 par les Bourbons de Naples, sanction alors couramment infligée aux opposants politiques dans l'Italie du XIXe siècle, y a indéniablement contribué ${ }^{1}$. Lors de l'unification de l'Italie, Poerio refuse plusieurs charges sénatoriales et ministérielles, occupe une simple charge de député, poste qu'il présente comme sa seule ambition politique, et réside à Florence, chez Ferdinando Fonseca, chez qui il décède en 1867. Outre sa pauvreté, ses thuriféraires évoquent sa

${ }^{39}$ Della vita e dei tempi di Carlo Poerio..., op. cit., p. 47.

40 ASCD, Discussioni, 29 avril 1867. Sur le modèle de la mort laïque, voir D. Mengozzi, Garibaldi taumaturgo..., op. cit., p. 133.

41 Aspect évoqué par Michele Pironti (ASCD, Discussioni, 29 avril 1867). Renata De Lorenzo, "Lotte politiche e patrimoni familiari. La sopravvivenza nella diaspora dagli esuli meridionali », Mélanges de l'École française de Rome. Italie et Méditerranée, 129-2, 2017. 
mansuétude à l'égard du régime de Ferdinand II et des populations de Naples, auxquelles il a présenté deux fois sa candidature à un siège législatif alors qu'une partie d'entre elles a contribué à sa condamnation à la prison, en 1849, au moyen de réseaux de faux témoins sur lesquels s'appuyait alors la justice bourbonienne ${ }^{42}$.

Ces qualités conduisent plusieurs orateurs à présenter Poerio comme transposant dans la société politique italienne des qualités christiques. Il apparaît alors comme une figure éminente de la sainteté laïque, notion qui s'est imposée dans les sociétés du XIX ${ }^{\mathrm{e}}$ siècle comme un outil légitimateur dont se dotent divers mouvements politiques pour s'appuyer sur des acteurs réputés exceptionnels. Amorcée parmi les mouvements démocratiques de la première moitié du XIX ${ }^{\mathrm{e}}$ siècle, qui ont commémoré des figures vertueuses de patriotes morts ou vivants, cette évolution s'est par la suite appliquée au discours officiel de l'Italie unie. Il fait de la sainteté l'un de ses piliers, aux côtés de la famille et de l'honneur ${ }^{43}$. Inscrit dans un panthéon de saints patriotiques à géométrie variable, Poerio apparaît doté d'une place éminente, régulièrement comparé au Christ dont il reproduirait l'action dans la sphère civile. Baldacchini rappelle ainsi qu'à son image, il s'est efforcé de pardonner à ses bourreaux, faisant montre d'une vertu et d'une abnégation largement mobilisées dans l'éthique du patriotisme italien depuis la fin du XVIIIe siècle ${ }^{44}$. De Simone, quant à lui, le définit comme entièrement voué à la cause patriotique, faisant preuve de modestie, de tolérance, soucieux du bien de ses semblables, y compris ses ennemis, et souligne qu'il « pardonnait à ses bourreaux, comme le Christ ${ }^{45}$ ». De tels parallèles reprennent une image courante dans les mouvements politiques du XIX ${ }^{\mathrm{e}}$ siècle, influencés par le contexte culturel du romantisme et par la recharge sacrale qui l'a accompagné, au point que la figure christique a été régulièrement mobilisée à la fois par les premiers socialismes et par l'évangélisme démocratique, notamment pendant les révolutions de $1848^{46}$. En Italie, elle doit ses principaux usages au succès du courant mazzinien qui a amplement recouru à la religiosité laïque comme outil intégrateur, construisant ainsi la sacralité de la construction nationale ${ }^{47}$. Ces figures catholiques sécularisées sont en partie récupérées dans la religion civile italienne. L'attribution de qualités christiques, qui relève directement de ces transferts, joue un rôle essentiel dans l'édification morale de Poerio. Alors qu'elle désigne plus couramment des abstractions ou des idéaux portés par des mouvements politiques, elle est utilisée comme un outil commémoratif, destiné à renforcer la sacralité de Carlo Poerio.

42 Della vita e dei tempi di Carlo Poerio..., op. cit., p. 3.

43 Alberto Mario Banti, La nazione del Risorgimento. Parentela, santità ed onore alle origini dell'Italia unita, Turin, Einaudi, 2000.

${ }^{44}$ Della vita e dei tempi di Carlo Poerio..., op. cit., p. 35. Sur les constructions morales dans lesquelles s'insère la virtù, voir Giulia Delogu, La poetica della virtù. Comunicazione e rappresentazione del potere in Italia tra Sette e Ottocento, Milan, Mimesis, 2018.

45 R. De Cesare, Carlo Poerio..., p. 8.

46 Voir sur ce point Frank Paul Bowman, Le Christ romantique, Genève, Droz, 1978.

${ }^{47}$ Simon Levis Sullam, « The Moses of Italian Unity. Mazzini and Nationalism as a Political Religion », dans Christopher A. Bayly, Eugenio F. Biagini (dir.), Giuseppe Mazzini and the Globalization of Democratic Nationalism, Oxford, Oxford University Press, 2008, p. 107124. 
$\mathrm{Au}$ titre de cette comparaison, plusieurs auteurs de nécrologes érigent le défunt en objet de dévotion, digne de recevoir un culte à la fois religieux et politique. Pironti, par exemple, définit à plusieurs reprises les hommages qui lui sont rendus comme les signes d'une «religion intime » qui «sanctifie la mort» de Poerio et lui assure une existence posthume, en tant que figure symbolique du panthéon national italien ${ }^{48}$. Les valeurs morales qui lui sont prêtées vont dans ce sens, en identifiant le patriote vertueux à un saint laïque. Du fait de sa capacité à souffrir, de la constance de son attachement à la cause nationale, plusieurs de ses thuriféraires lui reconnaissent une place supérieure parmi les martyrs de la liberté italienne et affirment son statut d'exemple, digne d'être imité par ses contemporains. Baldacchini le perçoit ainsi comme un « exemple divin », issu des " pages les plus suaves des Évangiles » au point de faire partie « de ceux que l'on doit croire » ${ }^{49}$. Pironti, quant à lui, appelle les Napolitains à « contempler ses vertus ${ }^{50}$ ». Ces invitations destinées au peuple italien rejoignent l'un des aspects du martyre politique du XIX siècle, qui tire de son substrat religieux originel une visée pédagogique fondamentale. Le maire de Naples Fedele De Siervo voit en Poerio une figure mobilisatrice, capable d'éduquer le peuple napolitain à la dignité. La continuité des expériences du martyre sert cette fonction : de la même manière qu'il aurait servi, pendant les années 1850, d'exemple de constance et d'abnégation à ses compagnons de cellule, les Napolitains peuvent le considérer comme un maître, auprès duquel apprendre l'attachement à la patrie ${ }^{51}$.

Les attestations nombreuses de cette identification montrent qu'à l'exemple des modèles religieux qu'il est supposé reproduire, la mort de Carlo Poerio est une mort utile, qui permet de consolider des communautés d'appartenance. Cet aspect de la mort patriotique, amplement développé dans le discours national italien ${ }^{52}$, transfère dans la société civile la fonction identitaire de la sainteté par rapport à des communautés territoriales définies par l'origine du martyr ou par le lieu de son action ${ }^{53}$. La ville de Naples, d'abord, fait valoir la mémoire de Poerio, à l'initiative du conseil communal. Celuici finance l'édition d'un opuscule commémoratif qui regroupe les obituaires prononcés en l'honneur du martyr, auxquels sont ajoutés les procès-verbaux qui ont accompagné son inhumation, présentés au lecteur dans les annexes du livre ${ }^{54}$. Dans la continuité d'un important effort porté à la patrimonialisation des figures fondatrices de la patrie par la construction des statues et la dénomination des voies publiques, le conseil communal de Naples renomme, une semaine après les obsèques de Poerio, une rue du quartier de Chiaia

\footnotetext{
48 Discours de Michele Pironti, 3 mai 1867, op. cit.

${ }^{49}$ Voir par exemple Della vita e dei tempi di Carlo Poerio..., op. cit., p. 3.

50 Discours de Michele Pironti, 3 mai 1867, op. cit.

51 Discours de Fedele De Siervo, 3 mai 1867, reproduit dans Carlo Poerio, op. cit., p. 10.

52 D. Mengozzi, Garibaldi taumaturgo..., op. cit., p. 64.

${ }^{53}$ Cet aspect a suscité d'importants questionnements de la part des sciences sociales. Voir notamment Pierre Centlivres (dir.), Saints, sainteté et martyre. La fabrication de l'exemplarité, Paris, Maison des Sciences de l'Homme, 2001, et Cyril Isnart, Saints légionnaires des Alpes du Sud. Ethnologie d'une sainteté locale, Paris, Maison des Sciences de l'Homme, 2008.

${ }^{54}$ Carlo Poerio, op. cit.
} 
en vico Carlo Poerio, décision approuvée à l'unanimité des présents ${ }^{55}$. Il prévoit par ailleurs l'érection d'un monument commémoratif, qu'il invite toutes les mairies d'Italie à cofinancer au moyen d'une souscription ${ }^{56}$. La même initiative prévoit l'édition ultérieure d'un recueil d'écrits et de lettres de Poerio, sur le modèle des "panthéons de papier » alors publiés dans les milieux patriotiques italiens ${ }^{57}$. Seul le projet de statue est suivi d'effets, aidé par le concours d'autres municipalités italiennes qui ont souscrit à sa construction. Installé au centre de la piazza Carità, il ancre la mémoire de Poerio dans l'espace public napolitain.

Les prétentions de la mairie de Naples à constituer le défunt en un saint laïque pleinement intégré à la mémoire locale trouvent des relais à Florence, dont l'État italien cherche à renforcer la fonction de capitale en inscrivant la mémoire publique de la nation dans l'espace urbain ${ }^{58}$. Alors que l'opinion florentine est particulièrement attentive au sort de la dépouille de Poerio - l'ample mobilisation du journal L'Opinione tout au long du mois de mars 1867 en témoigne -, plusieurs des oraisons funèbres prononcées en son honneur mettent en évidence son attachement pour la ville de Florence, où il a suivi son père en exil à deux reprises, en 1815 puis en $1823^{59}$. Le 10 mai 1867, le conseil communal de la ville envisage, sur la proposition du comte Fossombrone, alors l'un de ses membres, d'ériger une statue placée dans la basilique Santa Croce, auprès de celle consacrée deux ans plus tôt à une autre figure fondatrice du nouvel État italien, le Vénitien Daniele Manin ${ }^{60}$. Réalisée par le sculpteur florentin Giovanni Paganucci, elle est installée quelques mois plus tard, preuve du culte national rendu à Carlo Poerio. Les célébrations rendues à Florence relèvent en effet surtout d'une translation mémorielle, qui fait d'un martyr napolitain l'une des figures du panthéon national italien.

Les hommages rendus à Carlo Poerio révèlent donc son édification morale très rapide, destinée à faire valoir, dès les semaines qui ont suivi sa mort, le statut d'acteur d'exception et de figure fondatrice de la nation italienne qui lui est prêté. L'ample mobilisation des milieux parlementaires au service de sa mémoire montre la place des classes dirigeantes du nouvel État dans la consolidation d'une communauté nationale alors fragile autour d'une religion laïque à valeur intégratrice. Les commémorations font de son rôle de député l'une des étapes de son parcours de martyr, catégorie indéfinie et

55 ASMN, Deliberazioni, VIII, séance du 10 mai 1867, p. 16.

56 « A tutti i municipii d'Italia », Naples, 22 mai 1867, reproduite dans Carlo Poerio, op. cit., p. 113-115.

${ }^{57}$ Expression empruntée à Panteon dei martiri della libertà italiana, Turin, Fontana, 1851, vol. 1, p. 2.

58 Voir sur ce point Catherine Brice, «Lecture politique d'un espace urbain : Florence capitale, 1865-1870 », Mélanges de l'École française de Rome, 94/2, 1982, p. 847-889.

59 Outre Della vita e dei tempi di Carlo Poerio..., op. cit., p. 8, voir le discours d'Antonio Scialoja du 3 mai 1867, cité dans Carlo Poerio, op. cit., p. 5-9.

60 Archivio Storico Municipale di Firenze, Deliberazioni del Consiglio Comunale, 10 mai 1867. 
protéiforme qui associe un engagement moral, des caractères héroïques et une fonction de témoignage et qui constitue une prédisposition à la célébrité, alors que se constituent des panthéons de grands hommes. Pour autant, alors que la propagande du nouvel État italien fait valoir, jusqu'au XXe siècle, les figures fondatrices dont il est l'héritier, la mémoire de Carlo Poerio est assez rapidement éclipsée par celle d'autres membres de sa famille, notamment son frère Alessandro, mort en défendant Venise contre l'Autriche en 1848, dont l'œuvre poétique est amplement célébrée au travers d'hommages littéraires, de références politiques et de festivités collectives. À la mémoire éphémère de l'homme représentatif a donc succédé la postérité plus durable d'une «famille-emblème », pensée comme un collectif indissociable, que le discours national a érigé en modèle et en précurseur du patriotisme italien ${ }^{61}$.

61 Voir notamment Benedetto Croce, «Una famiglia di patrioti : I Poerio », La Critica. Rivista di Letteratura, Storia e Filosofia, 15, 1917, p. 1-15. 